\title{
Oscillatory Regularity of Charge Carrier Trap Energy Spectra in ZnSe Single Crystals
}

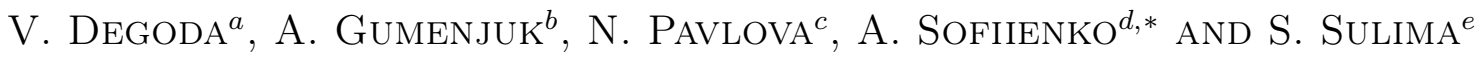 \\ ${ }^{a}$ Taras Shevchenko National University of Kyiv, Volodymyrs'ka 64, 01601 Kyiv, Ukraine \\ ${ }^{b}$ Institute of Physics of the National Academy of Science of Ukraine, Nauky ave. 46, 03028 Kyiv, Ukraine \\ ${ }^{c}$ National Pedagogical Dragomanov University, Pirogova 9, 01030 Kyiv, Ukraine \\ ${ }^{d}$ University of Bergen, Allegaten 55, PO Box 7803, 5020 Bergen, Norway \\ ${ }^{e}$ Institute for Single Crystals of the National Academy of Science of Ukraine, Lenin ave. 60, ${ }^{6} 1001$ Kharkiv, Ukraine
}

(Received April 23, 2015; in final form February 22, 2016)

This article presents the results of an experimental investigation of the energy spectra of charge carrier traps in undoped high-resistivity ZnSe single crystals. Fourteen peaks were found in the thermostimulated luminescence spectra of the ZnSe samples at temperatures between $8 \mathrm{~K}$ and $450 \mathrm{~K}$, and the thermal activation energies of the charge carrier traps were estimated for the most intense peaks. It was found that the energy spectra of the charge carrier traps in ZnSe exhibit oscillatory regularity, and the energy of a vibrational quantum was estimated to be $\hbar \omega=206 \mathrm{~cm}^{-1}$, which is in good agreement with the vibrational mode in the Raman spectrum. Additionally, a linear relationship was observed between the thermal activation energies of the charge carrier traps and the temperature positions of the maxima in the thermostimulated luminescence of ZnSe.

DOI: 10.12693/APhysPolA.129.304

PACS/topics: 64.70.kg, 68.55.ag, 07.77.Ka

\section{Introduction}

Charge carrier traps and defects in optical materials, particularly laser crystals and scintillators, do not always have a negative influence on the material's physical properties. Usually they define the intensity and spectra of the optically or radiation-stimulated luminescence. The deep traps in wide-band-gap materials utilized for thermoluminescence (TL) dosimetry [1] actually increase their sensitivity. Currently, monocrystalline zinc selenide $(\mathrm{ZnSe})$ is a prospective wide-band-gap semiconductor [2] for use as a detector of ionizing radiation $[3,4]$ at high temperatures of up to at least $130^{\circ} \mathrm{C}[3,5]$ due to its high resistivity and relatively low concentration of impurities and defects. ZnSe has a band gap of $2.8 \mathrm{eV}$ at $300 \mathrm{~K} \mathrm{[2]}$ and a high absorption efficiency for X-ray and gamma photons [6]. The physical features of its crystal structure allow single crystals to be grown in large volumes $\left(\Delta V>50 \mathrm{~cm}^{3}[7,8]\right)$, which is a significant advantage over other wide-band-gap semiconductors such as $\mathrm{SiC}$, GaN, InGaN or diamond $[9,10]$. Despite the possibility to grow large volumes of monocrystals of undoped $\mathrm{ZnSe}$, this material typically still has uncontrollable impurities conditioning the shallow and deep charge carrier traps, influencing the kinetics of charge carrier transport under gamma or X-ray excitation [11].

Primarily, charge carrier traps are investigated using thermostimulated luminescence (TSL). Although the investigations of the TSL of $\mathrm{ZnSe}$ have been performed

*corresponding author; e-mail: asofienko@gmail.com before [12-15], only doped crystals and crystals with high concentrations of uncontrollable impurities were investigated; the literature thus shows a lack of detailed information published about the TSL of undoped monocrystalline $\mathrm{ZnSe}$ and the energy spectra of charge carrier traps $(\mathrm{CCTs})$ in this semiconductor. To address the gap in knowledge mentioned above, the general purpose of this work is an investigation of the TSL of high-resistivity undoped ZnSe monocrystals $\left(\rho>10^{12} \Omega \mathrm{cm}\right)$ with a high optical quality to determine the regularity between the luminescence spectra of ZnSe and TSL peaks and to determine the existence of oscillatory regularity in the energy spectra of CCTs.

\section{Experimental section}

\subsection{Experimental methods}

ZnSe samples of $15 \times 10 \times 2 \mathrm{~mm}^{3}$ were cut from different undoped $\mathrm{ZnSe}$ monocrystals that were grown at the Institute for Single Crystals of NAS of Ukraine using the Bridgman method [16] and under an argon pressure of $15 \mathrm{~atm}$. The primary other selection criteria were the homogeneity and the high electrical resistance at room temperature $\left(\rho>10^{12} \Omega \mathrm{cm}\right)$. The crystal sides were mechanically polished in the final stage. Complex experimental research involving the X-ray luminescence (XRL) spectra at different temperatures (e.g., 8, 85, $295 \mathrm{~K}$ ), TSL spectra and phosphorescence $(\mathrm{Ph})$ in ZnSe single crystals were performed in a vacuum cryostat under low pressure $(P>1 \mathrm{~Pa})$. The samples were irradiated by the integral radiation of a BHV-7 X-ray tube (Re anode, $20 \mathrm{kV}$, $25 \mathrm{~mA}$, X-ray flux is $0.42 \mathrm{~mJ} /\left(\mathrm{s} \mathrm{cm}^{2}\right)$ in the sample plane) through a beryllium window. The luminescence and phosphorescence measurements were performed by 
two independent channels: integrally through an optical filter and spectrally through an MDR-2 monochromator at different wavelengths using a diffraction grating with 600 lines $/ \mathrm{mm}$. In the integral channel, all luminescent light is focused by a quartz lens onto the photocathode of a PMT-106 with a high spectral sensitivity between $350 \mathrm{~nm}$ and $820 \mathrm{~nm}$. Two PMTs were used for the spectral measurements: a PMT-106 and a PMT-83 with a high spectral sensitivity between $600 \mathrm{~nm}$ and $1200 \mathrm{~nm}$. All measured spectra were corrected based on the spectral sensitivity of the PMTs used. The investigations of $\mathrm{Ph}$ of $\mathrm{ZnSe}$ samples were performed after preliminary $\mathrm{X}$-ray irradiation for $20 \mathrm{~min}$ at different temperatures of 8,85 , and $295 \mathrm{~K}$. Ph was measured for 5 min after the X-ray irradiation and then the samples were heated with a constant heating rate of $\beta=0.3 \pm 0.02 \mathrm{~K} / \mathrm{s}$ $\left(T(t)=T_{0}+\beta t\right)$ to measure the TSL in all measuring channels within three temperature ranges: $8 \mathrm{~K}$ to $220 \mathrm{~K}, 85 \mathrm{~K}$ to $420 \mathrm{~K}$ and $295 \mathrm{~K}$ to $450 \mathrm{~K}$. The irradiation of the ZnSe samples at higher temperatures leads to an increase in the high-temperature peaks in the TSL due to additional intense charge accumulation in the deep traps. The heating of the samples was accomplished using an electrical heater integrated into the vacuum cryostat. The cooling of the samples was accomplished with liquid helium or nitrogen. Thermostimulated luminescence, which is usually intense in preliminarily cooled ZnSe crystals, is in fact a recombination radiation. The intensity of TSL, $J_{\mathrm{TSL}}$, on the beginning of the TSL peak is proportional to the speed of the decrease of the concentration of the filled traps, $n$, and to the Boltzmann factor as follows:

$$
\begin{gathered}
J_{\mathrm{TSL}} \sim-\frac{\mathrm{d} n(t)}{\mathrm{d} t}=-\frac{\mathrm{d} n}{\mathrm{~d} T} \frac{\mathrm{d} T}{\mathrm{~d} t}= \\
n(T) w_{0} \exp \left(-\frac{E_{\mathrm{T}}}{k T}\right),
\end{gathered}
$$

where $w_{0}$ is the frequency factor and $E_{\mathrm{T}}$ is the effective energy of the thermal delocalization of the charge carriers from the traps. The concentration of filled traps decreases with higher temperatures, while the Boltzmann factor increases. Therefore, the product of these two factors yields a curve with a maximum at some temperature. The derivative of formula (1) allows the calculation of this temperature's position, $T_{\mathrm{m}}$, at maximum of the TSL peak and the determination of the general relation for the frequency factor as follows:

$$
w_{0}=\frac{E_{\mathrm{T}} \beta}{k T_{\mathrm{m}}^{2}} \exp \left(\frac{E_{\mathrm{T}}}{k T_{\mathrm{m}}}\right),
$$

where $\beta=\mathrm{d} T / \mathrm{d} t$ is the heating rate of the sample. The energy spectrum of the traps was investigated using the method of fractional TSL (FTSL) [17]. This method is based on the assumption that the initial lowtemperature portion of the TSL peak can be well approximated by a simple exponential function because initial detrapping is a linear process for the first few percent change in accumulated charge. The sample is heated up until the TSL reaches of about $10 \%$ of the maximum intensity and then the sample is cooling down. This procedure is repeating several times and for each $N$-measurement the following assumption can be applied:

$$
J_{\mathrm{TSL}}(N, T)=J_{0 N} \exp \left(-\frac{E_{\mathrm{T}}}{k T}\right),
$$

where $J_{0 N}=w_{0 N} n \approx$ constant and the thermal delocalization energy (TDE), $E_{\mathrm{T}}$, can be found as an averaged value from the slopes of the experimentally measured curves (3) on a logarithmic scale: $E_{\mathrm{TN}}=k \times$ $\Delta \ln \left(J_{\mathrm{TSL}}(N, T)\right) / \Delta(1 / T)$. This procedure can be applied to each TSL peak. The described FTSL method has few advantages. First, the TDE can be computed using many measured FTSL-curves (3) for one selected TSL peak, and second, the existence of several plateaus on the experimentally determined dependence of the TDE upon the number of the cycle implies that there are CCTs with discrete energies [17].

\subsection{X-ray induced luminescence spectra of $\mathrm{ZnSe}$}

In general, the XRL spectra of $\mathrm{ZnSe}$ consist of edge excitonic emission (EEE), emissions of donor-acceptor pairs (DAPs) and wide recombination emission bands with maxima at 635, 820, and $970 \mathrm{~nm}$. For example, the XRL spectra of four ZnSe samples measured at liquid nitrogen temperature are shown in Fig. 1. In the

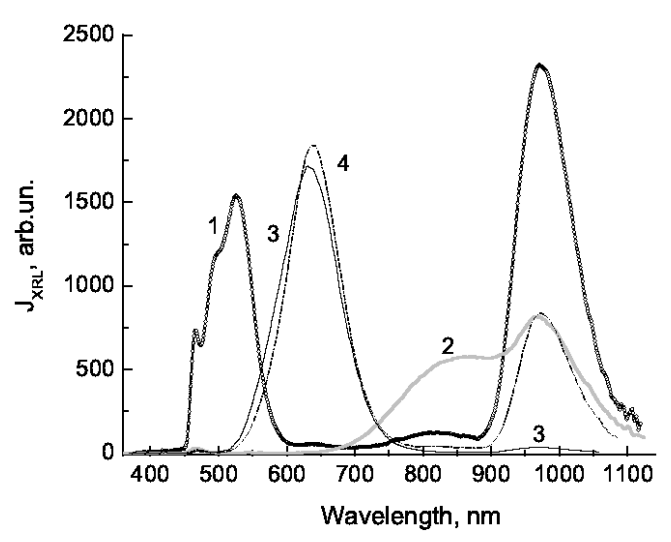

Fig. 1. XRL spectra of four ZnSe samples measured at $85 \mathrm{~K}$.

remaining ZnSe crystals, the same XRL bands were measured but with different intensities. The XRL spectra measured at liquid helium temperature are nearly identical. A significant change in the XRL spectra is observed at room temperature due to temperature quenching of the luminescence. The typical EEE is observed in all studied ZnSe samples at low temperatures [14, 18, 19]; this emission is detected as a band with a maximum at $465 \mathrm{~nm}$ at liquid nitrogen temperature. EEE is conditioned by the excitons that are free or localized on the shallow donors [20, 21]. This emission is intensive at liquid helium temperature and rapidly quenched after heating with a characteristic energy of $50 \mathrm{meV}$, which is the dissociation energy of the excitons in $\mathrm{ZnSe}$ [22]. 
In the ZnSe crystals in which the recombination emission bands with maxima at $635 \mathrm{~nm}$ and $820 \mathrm{~nm}$ are weak, the intensive emission of DAPs is usually observed $[18,23,24]$ in the wavelength range of $470 \mathrm{~nm}$ to $550 \mathrm{~nm}$. The energy levels of the donors in ZnSe are conditioned by the $\mathrm{Zn}_{\mathrm{i}}$ defects, and the acceptor centres may be conditioned by $\mathrm{O}_{\mathrm{Se}}$ or $\left(\mathrm{O}_{\mathrm{Se}}-\mathrm{D}\right)$. The features of the luminescence of the DAPs have been studied in several works [23-26]. In undoped ZnSe crystals, a wide emission band with a maximum at $490 \mathrm{~nm}$ is observed as well. This emission band may be conditioned by the isolated oxygen centres $\mathrm{O}_{\mathrm{Se}}[27]$. Wide recombination bands with maxima at 635,820 , and $970 \mathrm{~nm}$ are known from the previous studies of ZnSe [13, 14, 27-31]. The emission band at $635 \mathrm{~nm}$, in accordance with [27, 29], can be associated with a complex centre consisting of oxygen at the lattice point and zinc vacancies $\mathrm{V}_{\mathrm{Zn}}$ and $\mathrm{Zn}_{\mathrm{i}}$. The emission of ZnSe in the infrared range from $820 \mathrm{~nm}$ to $970 \mathrm{~nm}$ is probably conditioned by a selenium vacancy [28] at the lattice point, and in accordance with $[30,31]$, the emission band at $970 \mathrm{~nm}$ is conditioned by a complex centre consisting of a selenium vacancy or a copper impurity. It should be noted that the spectral position of the emission band at $635 \mathrm{~nm}$ is different in different $\mathrm{ZnSe}$ samples, which indicates the non-elementary nature of this band [14], which may consist of at least two emission subbands, the difference in the spectral position of which is less than their half-width.

\subsection{Thermostimulated luminescence of $\mathrm{ZnSe}$}

The TSL curves of the ZnSe samples were measured in integral and spectral measuring channels at different luminescence band maxima of 635, 820, and $970 \mathrm{~nm}$. The same TSL peaks were detected in each case, but the intensities of the peaks were different in each sample. It should be noted that TSL peaks in ZnSe have a greater half-width $(\delta)$ than those in many other oxide crystals (e.g., $\delta \approx 20 \mathrm{~K}$ for the TSL peak at $T_{\mathrm{m}}=183 \mathrm{~K}$ ), which leads to an overlap of adjacent peaks. Figure 2 shows the measured TSL curves of several ZnSe samples that have different XRL spectra. Temperature luminescence quenching of the luminescence bands in $\mathrm{ZnSe}$ is observed above $220 \mathrm{~K}$. Therefore, the intensity and the temperature position of the primary TSL peaks do not change significantly despite the change in the quantum yield of luminescence with temperature.

The TSL in ZnSe is observed also at temperatures below $85 \mathrm{~K}$. Figure 3 shows two TSL peaks of different ZnSe samples measured after preliminary X-ray irradiation at $8 \mathrm{~K}$. It was found that low-temperature TSL in the temperature range of $8 \mathrm{~K}$ to $85 \mathrm{~K}$ is intensive, which indicates a high concentration of shallow traps in the samples. The low-energy tail of the TSL of sample 3 (curve 3 in Fig. 3) was used to estimate the delocalization energy of the appropriate traps.

It should be noted that there is no single value correlation between the intensities of the TSL peaks and the luminescence bands in XRL spectra. From experimental

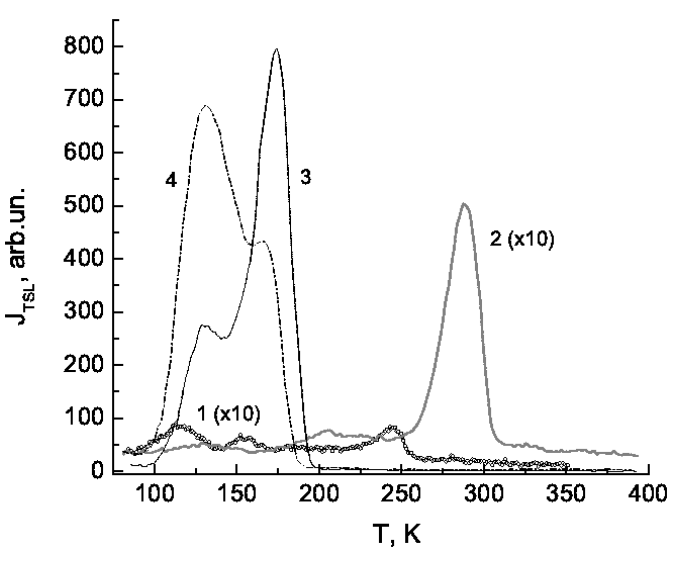

Fig. 2. TSL curves of four ZnSe samples (1-4) measured after preliminary X-ray irradiation for $20 \mathrm{~min}$ at $85 \mathrm{~K}$. The numbers are the same as shown in Fig. 1.

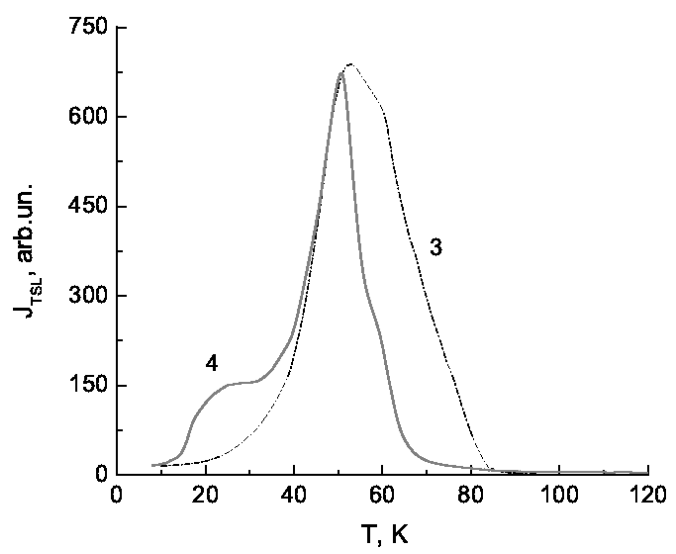

Fig. 3. Measured TSL curves of two ZnSe samples (samples 3 and 4) irradiated preliminarily by X-rays at $8 \mathrm{~K}$ for $20 \mathrm{~min}$.

observations, the samples with intensive XRL at $635 \mathrm{~nm}$ usually have an intense TSL peak at $T_{\mathrm{m}}=170 \mathrm{~K}$, and samples with intensive XRL at $820 \mathrm{~nm}$ usually have an intense TSL peak at $T_{\mathrm{m}}=279 \mathrm{~K}$. The XRL band at $970 \mathrm{~nm}$ shows no correlation with the TSL curves.

\section{Results and discussion}

Earlier experimental studies of the energy spectra of CCTs in many ion-covalent crystals (primarily oxides and alkali halides) using TSL methods [17, 32-39] showed that the energy spectra of CCTs are well described by the harmonic oscillator formula:

$$
E=\hbar \omega\left(n+\frac{1}{2}\right),
$$

where $n=1,2,3 \ldots$ is the vibrational quantum number and $\hbar \omega$ is the energy of the vibrational quantum. Several oscillatory regularities with different values of the energy of a vibrational quantum can be found in compound crystals. For example, one oscillatory regularity was found in sapphire [32], two in caesium cadmium trichloride [33], three in zinc tungstate and cadmium tungstate [34, 35], 
and five in yttrium aluminium garnet [36]. It is important that the energies of vibrational quanta in oscillatory regularities found for these materials coincide with symmetrical vibrational modes, $A_{g}$, that are active in the Raman spectra. Only single-series sequences exist in such alkali-halide crystals as $\mathrm{NaCl}$ [17], $\mathrm{LiF}$ [37], $\mathrm{KBr}$ [38], $\mathrm{KCl}$ and $\mathrm{NaI}$ [39]. Such crystals with simple cubic lattices do not have vibrational modes that are active in the Raman spectrum, and the frequencies of their oscillatory regularities correspond to the local oscillations of the H-centres. The modern explanation of oscillatory regularities in the energy spectra of CCTs is based on an assumption about their polaron nature [37]. The most intense peaks in the measured TSL curves of the investigated ZnSe samples were used to estimate the thermal activation energies (TAEs) of the traps using the FTSL method.

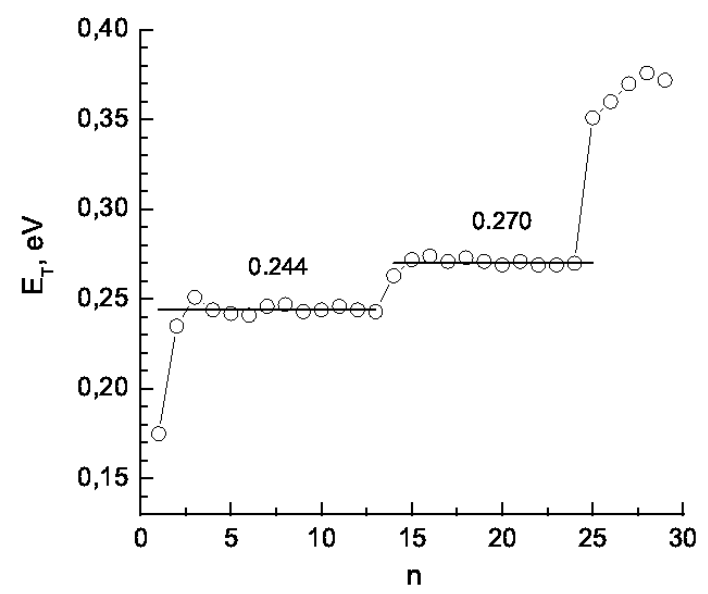

Fig. 4. Dependence of the computed TAE on the number of fraction in the processed TSL curves.

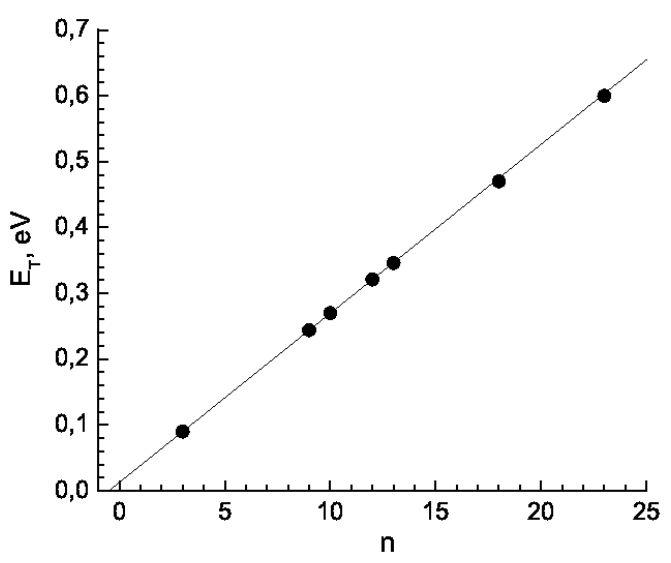

Fig. 5. Oscillatory regularity of the TAEs of CCTs in the studied ZnSe samples.

Figure 4 shows the results of the computation of the TAEs for all fractions in the processed TSL curves. The two plateaus in Fig. 4 with levels of $0.244 \pm 0.002 \mathrm{eV}$ and $0.270 \pm 0.002 \mathrm{eV}$ indicate that at least two groups of CCTs exist in the investigated ZnSe samples. The analysis of the obtained results shows that the found values of TAEs of traps are described well by oscillatory regularity (4) with the energy of the vibrational quantum $\hbar \omega=0.02567 \pm 0.0001 \mathrm{eV}\left(206 \pm 1 \mathrm{~cm}^{-1}\right)$.

This oscillatory regularity is shown in Fig. 5 as a function of the vibrational quantum number $n$. It was found that the vibrational quantum frequency is equal to the frequency of an optical transverse mode in $\mathrm{ZnSe}$, which is $207 \mathrm{~cm}^{-1}$ [40-42].

It was also found that the temperature positions of the maxima of all measured TSL peaks are well described by oscillatory regularity as follows:

$$
T_{\mathrm{m}}=\left(n+\frac{1}{2}\right) \Delta T[\mathrm{~K}]
$$

where $n=1,2,3 \ldots$ are integer numbers and $\Delta T=$ $13.55 \pm 1 \mathrm{~K}$. Function (5) is shown in Fig. 6 for 14 measured TSL peaks. The proportional relationship between the TAE of CCTs and the temperature position of the appropriate peak in the TSL can be found from Eqs. (4) and (5):

$$
E_{\mathrm{T}}=\frac{\hbar \omega}{k \Delta T} k T_{\mathrm{m}}=A k T_{\mathrm{m}}
$$

where $A=21.9$ for $\mathrm{ZnSe}$ at a constant heating rate of $\beta=0.3 \mathrm{~K} / \mathrm{s}$.

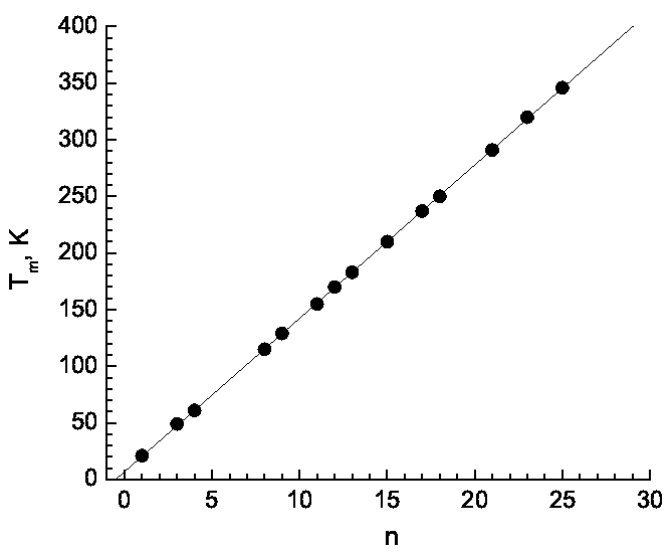

Fig. 6. Oscillatory regularity of the peaks in the TSL spectra of the studied ZnSe samples.

As shown in formula (3), the $T_{\mathrm{m}}$ value depends on the heating rate $\beta$ of the sample. The proportionality between $E_{\mathrm{T}}$ and $T_{\mathrm{m}}$ shown in formula (6) is known for the crystal phosphors [43, 44] and is characterized by one non-dimensional constant $A$. Formula (6) is usually used for a rough estimation of the TAEs of CCTs using the temperature positions of the peaks in the TSL. It is thus necessary to estimate the constant $A$ for any separate TSL peak using, for example, the FTSL method. The existence of oscillatory dependence (4) for all intensive maxima in the TSL of the studied ZnSe samples indicates that other low-intensive peaks that cannot be processed by the FTSL method also may follow this oscillatory dependence. This assumption allows the computation of the frequency factors for all traps in the studied samples as follows: 


$$
w_{0}=A \frac{\beta}{T_{\mathrm{m}}} \exp (A) .
$$

Because $A$ and $\beta$ are constants, then for any $i$-trap, the following condition is valid: $w_{0 i}=1 / T_{m i}$; the frequency factor is also monotonically decreasing when more deep traps are present. This indicates that point defects in the crystal are caused by the stabilization of a polaron in the crystal lattice at some energy level. The important fact that confirms the existence of the oscillatory regularity in the energy spectrum of CCTs of ZnSe is the coincidence of the energy of the vibrational quantum (4) of this dependence $\hbar \omega=0.0256 \mathrm{eV}\left(206 \mathrm{~cm}^{-1}\right)$ with a vibrational mode in the Raman spectrum $v=207 \mathrm{~cm}^{-1}$ that belongs to the symmetrical vibrational mode of the ZnSe crystal lattice [40-42].

Usually, free charge carriers polarize the nearest ions in the crystals with an ion-covalent bond, meaning that they are in a polaron state. Therefore, a filled trap can be presented as a polaron stabilized by the electric field of some defect. For this reason, the interaction of the polaron with phonon modes of the same symmetry $A_{g}$ is necessary to release the charge carriers from the potential well created by the polarization of the crystal lattice. This polarization distortion consists of a static shift of ambient charges in a line of excess charge. In the case of a symmetrical vibrational mode when the vibrations conserve the inversion operation, the charges also vibrate in the line of one centre. Therefore, for some phase of the vibration at a sufficient amplitude (or at a sufficient number of the absorbed phonons), the spatial positions of the ions may coincide with the lattice points in an ideal crystal and compensate for the field of the potential well created by the polarization of the crystal lattice. Under such conditions, there is a possibility of detrapping localized charge carriers.

It was found that the singularity of the interaction of the polaron with the crystal lattice defines the existence of traps with energies that are multiples of an integer $[37,38]$ or half-integer value $[32-36]$ of the energy of vibrational quantum $\hbar \omega$. The normalized probability of a polaron in the vibrational mode with energy $E=\hbar \omega\left(n+\frac{1}{2}\right)$ is described by the following:

$$
w_{n}=\frac{g_{n}}{S} \exp \left(-\frac{\hbar \omega(n+1 / 2)}{k T}\right),
$$

where $n$ is the vibrational quantum number, $S$ is the statistical sum that normalizes function (8) on a unit area and $g_{n}$ is the statistical weight. If only an oscillatory mechanism of the transition of free charge carriers to the recombination centres is present (e.g., due to tunnelling) without transition to the conduction band, we get the following relation for the statistical sum considering that $\sum_{n} w_{n}=1$ :

$$
S=\exp \left(-\frac{\hbar \omega}{2 k T}\right) \frac{1-\exp \left(-(n+1) \frac{\hbar \omega}{k T}\right)}{1-\exp \left(-\frac{\hbar \omega}{k T}\right)} .
$$

For $\hbar \omega=0.0256 \mathrm{eV}$ and $T>10 \mathrm{~K}$, we have $\exp (-\hbar \omega / k T) \ll 1$, and

$$
w_{n} \sim \exp \left(-\frac{N \hbar \omega}{k T}\right) .
$$

In this case, the energy of the traps is a multiple of an integer number of the vibrational quanta. If charge carriers transition into the conduction band after delocalization from the traps, the final state of the oscillator will consist of the states in the conduction band, and in this case, the statistical sum consists of both the oscillatory and band contributions: $S=S_{\text {osc }}+S_{\text {band }}$. As estimated, the band component is larger in fewer orders than the oscillatory component due to its significant statistical weight. As a result, the oscillatory component can be neglected, and finally, the energies of the traps depend on the half-integer value of the energy of vibrational quantum

$$
w_{n} \sim \exp \left(-\frac{(n+1 / 2) \hbar \omega}{k T}\right) .
$$

This conclusion indicates that the charge migration to the recombination centres occurs in part due to the collective states of the crystal lattice throughout the energy band. If the energies of the traps depend on the integer value of the energy of vibrational quantum (10), charge carriers can then directly tunnel from the traps to nearby recombination centres. In $\mathrm{ZnSe}$, the dependence of the energies of traps on the half-integer value of the energy of the vibrational quantum was found experimentally and confirms the existence of only one mechanism of charge migration from the traps to the recombination centres through the energy band.

\section{Conclusions}

We investigated the energy spectrum of charge carrier traps in undoped highly resistive $\mathrm{ZnSe}$ single crystals. Fourteen peaks were found in the thermostimulated luminescence spectra of ZnSe samples at temperatures from $8 \mathrm{~K}$ to $450 \mathrm{~K}$, and the energies of the thermal delocalization of the charge carriers for the appropriate groups of traps were estimated for the most intensive peaks. It was found that the energy spectrum of traps in undoped monocrystalline ZnSe follows oscillatory regularity, and the energy of vibrational quantum was estimated to be $\hbar \omega=206 \pm 1 \mathrm{~cm}^{-1}$, which is in good agreement with a vibrational mode in the Raman spectrum $\nu=207 \mathrm{~cm}^{-1}$. Additionally, a linear relationship is observed between the thermal activation energies of the traps and the temperature position of the peaks in the thermostimulated luminescence of $\mathrm{ZnSe}$. It was found experimentally that the energies of the charge carrier traps in ZnSe depend on the half-integer value of the energy of vibrational quantum; this result confirms the existence of only one mechanism of charge migration from the traps to the recombination centres through the energy band.

\section{Acknowledgments}

This work was partially funded by the Taras Shevchenko National University of Kyiv. All test samples were manufactured by Scientific \& Production Centre "Arvina" Ltd. (Ukraine). 


\section{References}

[1] V. Kortov, Radiat. Meas. 42, 576 (2007).

[2] M. Levinstein, S. Rumyantsev, M. Shur, Handbook Series on Semiconductor Parameters, Vol. 1,2, World Sci., London 1996, p. 1999.

[3] A.O. Sofiienko, V.Ya. Degoda, Radiat. Meas. 47, 27 (2012).

[4] K. Katrunov, V. Ryzhikov, V. Gavrilyuk, S. Naydenov, O. Lysetska, V. Litichevskyi, Nucl. Instrum. Methods Phys. Res. A 712, 126 (2013).

[5] M.S. Brodin, V.Ya. Degoda, A.O. Sofiienko, B.V. Kozhushko, V.T. Vesna, Radiat. Meas. 65, 36 (2014).

[6] J.H. Hubbell Int. J Appl. Radiat. Isot. 33, 1269 (1996).

[7] S. Fujiwara, H. Morishita, T. Kotani, K. Matsumoto, T. Shirakawa, J. Cryst. Growth 186, 60 (1998).

[8] Yu.V. Korostelin, V.I. Kozlovsky, A.S. Nasibov, P.V. Shapkin, J. Cryst. Growth 197, 449 (1999).

[9] A. Owens, A. Barnes, R.A. Farley, M. Germain, P.J. Sellin, Nucl. Instrum. Methods Phys. Res. A 695, 303 (2012).

[10] S.W. Rainer, Nucl. Instrum. Methods Phys. Res. A 582, 824 (2007).

[11] V.Ya. Degoda, A.O. Sofiienko, Physica B Condens. Matter 426, 24 (2013).

[12] D.D. Nedeoglo, A.V. Simashkevich, Electrical and Luminescence Properties of Zinc Selenide, Shtiintsa, Kishinev 1984, p. 150 (in Russian).

[13] G. Hitier, D. Curie, R. Visocekas, J. Phys. France 42, 479 (1981).

[14] I. Dafinei, M. Fasoli, F. Ferroni, E. Mihóková, F. Orio, S. Pirro, A. Vedda, IEEE Trans. Nucl. Sci. 57, 1470 (2010).

[15] H.L. Oczkowski, Acta Phys. Pol. A 82, 367 (1992).

[16] V.K. Komar, D.P. Nalivaiko, S.V. Sulima, Funct. Mater. 16, 192 (2009).

[17] A.F. Gumenjuk, S.Y. Kutovyi, Centr. Europ. J. Phys. 1, 307 (2003).

[18] K. Karpinska, A. Suchocki, M. Godlewski, D. Hommel, Acta Phys. Pol. A 84, 551 (1993).

[19] E. Tournie, C. Morhain, Appl. Phys. Lett. 68, 1356 (1996).

[20] J.C. Bouley, P. Blanconnier, A. Herman, J. Appl. Phys. 46, 3549 (1975).

[21] Yu.F. Vaksman, Yu.A. Nitsuk, Yu.N. Purtov, P.V. Shapkin, Semiconductors 35, 883 (2001).

[22] A.A. Artamonova, V.Ya. Degoda, V.E. Rodionov, Proc. SPIE 2113, 98 (1993).
[23] M.A.J. Klik, T. Gregorkiewicz, I.N. Yassievich, V.Yu. Ivanov, M. Godlewski, Phys. Rev. B 72, 125205 (2005).

[24] V. Ryzhikov, P. Gashin, N. Starzhinskiy, Funct. Mater. 10, 207 (2003).

[25] P. Bäume, S. Strauf, J. Gutowski, M. Behringer, D. Hommel, J. Cryst. Growth 184-185, 531 (1998).

[26] P. Bäume, J. Gutowski, D. Wiesmann, R. Heitz, A. Hoffmann, E. Kurtz, D. Hommel, G. Landwehr, Appl. Phys. Lett. 67, 1914 (1995).

[27] N.K. Morozova, I.A. Karetnikov, E.M. Gavrishchuk, Inorg. Mater. 35, 917 (1999) (in Russian).

[28] F.J. Bryant, P.S. Manning, J. Phys. 5, 1914 (1972).

[29] N.K. Morozova, V.A. Kuznetsov, V.D. Ryzhykov, Zinc Selenide. Production and Optical Properties, Nauka, Moscow 1992, p. 93 (in Russian).

[30] N.K. Morozova, I.A. Karetnikov, V.V. Blinov, E.M. Gavrishchuk, Semiconductors 35, 512 (2001).

[31] N.K. Morozova, I.A. Karetnikov, V.V. Blinov, E.M. Gavrishchuk, Semiconductors 35, 24 (2001).

[32] A.F. Gumenjuk, M. Grebenovych, S.J. Kutovyi, Funct. Mater. 9, 314 (2002).

[33] G.P. Blinnikov, V.M. Holonchka, A.A. Gumenjuk, Opt. Spectrosc. 69, 1054 (1990).

[34] I.S. Gorban, A.F. Gumenjuk, S.Y. Kutovyi, Ukr. J. Phys. 40, 73 (1995).

[35] A.F. Gumenjuk, O.B. Ochrimenko, S.Y. Kutovyi, Ukr. J. Phys. 42, 870 (1997).

[36] I.S. Gorban, A.F. Gumenjuk, V.Ya. Degoda, S.Y. Kutovyi, Opt. Spectrosc. 75, 47 (1993) (in Russian).

[37] A.F. Gumenjuk, S.Y. Kutovyi, Ukr. J. Phys. 50, 1125 (2005)

[38] A.F. Gumenjuk, S.Y. Kutovyi, O. Stanovyy, Visn. KNU Shevchenko, Ser. Fiz. 10-11, 32 (2010) (in Ukrainian).

[39] A.F. Gumenjuk, S.Y. Kutovyi, V.G. Pashchenko, O.P. Stanovyi, Ukr. J. Phys. 54, 999 (2009).

[40] U.A. Vinogradova, B.N. Mavrin, L.K. Voopjanov, J. Exp. Theor. Phys. 126/4, 866 (2004) (in Russian).

[41] S. Anand, P. Verma, K.P. Jain, S.C. Abbi, Physica B Condens. Matter 226, 331 (1996).

[42] K. Nakano, P.J. Boyce, J.J. Davies, D. Wolverson, J. Cryst. Growth 117, 331 (1992).

[43] A.M. Gurvich, X-Ray Phosphors and X-Ray Screens, Atomizdat, Moscow 1976, p. 152 (in Russian).

[44] A.M. Gurvich, Introduction to the Physical Chemistry of Crystal Phosphors, Vysshaya Shkola, Moscow 1982, p. 376 (in Russian). 